Research Article

\title{
Bioserotypes, Virulence Markers, and Antimicrobial Susceptibility of Yersinia enterocolitica Strains Isolated from Free-Living Birds
}

\author{
Marta Odyniec, ${ }^{1}$ Tomasz Stenzel, ${ }^{2}$ Dorota Lawreszuk, ${ }^{3}$ and Agata Bancerz-Kisiel $\mathbb{D}^{1}$ \\ ${ }^{1}$ Department of Epizootiology, Faculty of Veterinary Medicine, University of Warmia and Mazury in Olsztyn, Poland \\ ${ }^{2}$ Department of Poultry Diseases, Faculty of Veterinary Medicine, University of Warmia and Mazury in Olsztyn, Poland \\ ${ }^{3}$ Faculty of Biology and Chemistry, Institute of Biology, University of Białystok, Poland \\ Correspondence should be addressed to Agata Bancerz-Kisiel; a.bancerz-kisiel@uwm.edu.pl
}

Received 21 November 2019; Revised 28 February 2020; Accepted 5 March 2020; Published 17 March 2020

Academic Editor: Pengjun Shi

Copyright ( 2020 Marta Odyniec et al. This is an open access article distributed under the Creative Commons Attribution License, which permits unrestricted use, distribution, and reproduction in any medium, provided the original work is properly cited.

\begin{abstract}
The risk of meat contamination with Yersinia enterocolitica poses a threat to consumers and persons who come into contact with bird carcasses. The occurrence of Y. enterocolitica in the vast majority of migratory game species, the capercaillie, and the black grouse has never been studied in Poland, Europe, or in the world. The material for the study consisted of cloacal swabs obtained from 143 Eurasian coots, 50 mallards, 30 pochards, 27 greylag geese, 22 white-fronted geese, 22 bean geese, 20 green-winged teals, and 10 tufted ducks, as well as fecal swabs obtained from 105 capercaillie and 18 black grouse. Bacteriological examinations of 894 samples taken from 447 birds led to the isolation of 20 strains with the biochemical features characteristic of the genus Yersinia. All 20 strains were molecularly examined, and the genes characteristic of Y. enterocolitica were detected in 8 strains. The isolated strains harbored amplicons whose size corresponded to $y s t B$ gene fragments. Four strains belonged to bioserotype 1A/NI, one strain was identified as bioserotype 1B/O:9, and one as 1A/O:9. The prevalence of Y. enterocolitica was determined at $1.4 \%$ in green-winged teals, at $5.0 \%$ in Eurasian coots, and at $4.8 \%$ in capercaillie. All strains were resistant to amoxicillin with clavulanic acid, ampicillin, and cefalexin. The strains isolated from migratory birds were also resistant to kanamycin and streptomycin, and they were characterized by resistance or intermediate resistance to cefotaxime, ceftazidime, chloramphenicol, gentamycin, and tetracycline, to which the strains isolated from the capercaillie were susceptible. Yersinia enterocolitica was not detected in the remaining bird species. The presence of Y. enterocolitica in green-winged teals, Eurasian coots, and capercaillie indicates that these birds could be carriers, potential reservoirs, and sources of infection for humans. They can also be regarded as reliable bioindicators of Y. enterocolitica in their respective habitats.
\end{abstract}

\section{Introduction}

Free-living birds are a highly interesting and insufficiently investigated group of animals. Game birds play an important role in public health as a potential source of infection with pathogens dangerous for human health. In Poland, 13 species of free-living birds can be hunted: pheasant (Phasianus colchicus), green-winged teal (Anas crecca), tufted duck (Aythya fuligula), greylag goose (Anser anser), white-fronted goose (Anser albifrons), bean goose (Anser fabalis), pochard (Aythya ferina), wood pigeon (Columba palumbus), hazel grouse (Tetrastes bonasia), mallard (Anas platyrhynchos), partridge (Perdix perdix), Eurasian coot (Fulica atra), and Eurasian woodcock (Scolopax rusticola). Most of these birds are migratory species that travel considerable distances during migrations and use natural water resources, which underscores their importance in the epidemiological context. The capercaillie (Tetrao urogallus) and the black grouse (Tetrao tetrix) are classified as endangered species in Poland [1]. However, despite the progressing decrease in the size of capercaillie and black grouse populations, these species are regarded as game birds in some countries (e.g., Belarus, 
Russia, Sweden, Finland, and Norway) and are hunted for meat and trophies [1]. Unlike other game birds, the capercaillie and the black grouse do not migrate and inhabit specific territories.

The genus Yersinia of the family Enterobacteriaceae is composed of 19 species, three of which (Y.pestis, Y. enterocolitica, and $Y$. pseudotuberculosis) are pathogenic for humans and animals $[2,3]$. Although yersiniosis is not highly prevalent and dangerous for animals, animals are often carriers and sources of Yersinia infections for humans. In Europe, $Y$. enterocolitica is the most important etiological factor of human yersiniosis, whereas $Y$. pseudotuberculosis infections rarely cause yersiniosis [4]. Raw or undercooked pork is the main source of human $Y$. enterocolitica infections, and pigs are the main reservoir of bacteria $[5,6]$. However, Y. enterocolitica was also detected in various species of wild animals. In Poland, the pathogen has been identified in wild boars, roe deer, red deer, and fallow deer [7-9]. In the group of game birds, only pheasants and mallards have been tested for the presence of Y. enterocolitica in Poland [10]. The risk of meat contamination with $Y$. enterocolitica poses a threat to consumers and persons who come into contact with bird carcasses. The occurrence of $Y$. enterocolitica in the vast majority of migratory game species, the capercaillie, and the black grouse has never been studied in Poland, Europe, or in the world.

Yersinia enterocolitica strains are classified into six biotypes (1A, 1B, and 2-5) and more than 70 serotypes. Pathogenic strains harbor the Yersinia virulence plasmid (pYV) and ail and $y s t A$ chromosomal genes which encode the production of attachment invasion locus (Ail) protein and Yersinia-stable toxin A (YstA), respectively [2]. Biotype 1A strains that do not harbor pYV and ail and ystA genes are usually considered nonpathogenic $[2,11,12]$. However, in recent years, Y. enterocolitica biotype $1 \mathrm{~A}$ strains have also been isolated from clinical cases of yersiniosis presenting with diarrhea $[6,13,14]$. Although biotype $1 \mathrm{~A}$ strains very rarely produce YstA enterotoxin, they usually harbor the $y s t B$ gene which encodes the production of YstB enterotoxin. The minimal lethal dose of YstB is much lower in comparison with YstA enterotoxin $[15,16]$. Extremely rare strains of biotype $1 \mathrm{~A}$ harbor the $y s t C$ gene which encodes the production of YstC enterotoxin [16], and they have never been isolated from clinical cases of yersiniosis.

The aim of this study was to identify the bioserotypes, virulence markers, and antimicrobial susceptibility of $Y$. enterocolitica strains isolated from the different species of game birds in Poland, as well as from the capercaillie and black grouse.

\section{Materials and Methods}

2.1. Materials. The material for the study consisted of 286 cloacal swabs obtained from 143 Eurasian coots, 100 cloacal swabs obtained from 50 mallards, 60 cloacal swabs obtained from 30 pochards, 54 cloacal swabs obtained from 27 greylag geese, 44 cloacal swabs obtained from 22 white-fronted geese, 44 cloacal swabs obtained from 22 bean geese, 40 cloacal swabs obtained from 20 green-winged teals, and 20 cloacal swabs obtained from 10 tufted ducks. Two samples were collected from each bird and cultured in two different media. The samples from free-living birds were collected during the hunting seasons specific for each species, between autumn 2016 and spring 2019, in hunting districts throughout Poland.

Two hundred and ten fecal swabs from 105 capercaillie fecal samples and 36 fecal swabs from 18 black grouse fecal samples were also collected. Most capercaillie were kept as a part of the EU LIFE Project entitled "Active protection of lowland populations of capercaillie in the Bory Dolnośląskie and the Augustowska Primeval Forest", authorization no. DZP-WG.6401.03.100.2016.km of 31 May 2016, granted by the General Directorate of Environmental Protection. Some samples were collected from wild capercaillie in the Kirov region (Russia) and the Wildlife Park in Kadzidłowo (Poland). All samples were collected from aviaries in breeding centers in 2016-2017. Black grouse samples were collected in June 2017 in the Jedwabno State Forest District which features 5 black grouse preservation zones.

2.2. Biochemical Identification. A total of 894 swabs collected from 447 birds were examined. One swab was placed on the ITC (irgasan, ticarcillin, and potassium chlorate-warm culture) medium, and the other swab was placed on the PSB (peptone, sorbitol, and bile salts-cold culture) medium. Swabs on ITC were incubated at $25^{\circ} \mathrm{C}$ for $48 \mathrm{~h}$, and swabs on PSB were incubated at $4^{\circ} \mathrm{C}$ for 3 weeks to determine the ability of Yersinia to grow at low temperatures. Next, $0.5 \mathrm{ml}$ of each culture was transferred to $4.5 \mathrm{ml}$ of $0.5 \% \mathrm{KOH}$ in $0.5 \% \mathrm{NaCl}$ for $20 \mathrm{~s}$, and a loopful was streaked onto a CIN (cefsulodin, irgasan, and novobiocin) plate and incubated at $30^{\circ} \mathrm{C}$ for $48 \mathrm{~h}$. Colonies typical for Yersinia were transferred to MacConkey agar, incubated at $30^{\circ} \mathrm{C}$ for $24 \mathrm{~h}$ and biochemically analyzed using API 20E strips (bioMérieux, France) according to the manufacturer's instructions.

2.3. DNA Isolation. Genomic DNA was isolated with the Genomic Mini Kit (A\&A Biotechnology, Gdynia, Poland) according to the manufacturer's instructions. DNA was stored at $-20^{\circ} \mathrm{C}$ for further analyses.

2.4. Molecular Confirmation of Y. enterocolitica Strains. Four chromosomal genes-ail, yst $A, y s t B$, and $y s t C$-were amplified in one reaction. The primers for ail, ystA, ystB, and $y s t C$ were described previously [7, 17]. Multiplex PCR was performed using HotStarTaq Plus DNA Polymerase (Qiagen $\mathrm{GmbH}$, Hilden, Germany) and HotStarTaq Plus Master Mix Kit (Qiagen). The reaction mixture of $20 \mu \mathrm{l}$ contained approximately $120 \mathrm{ng}$ of isolated DNA ( 1 to $3 \mu \mathrm{l}$ ), $10 \mu \mathrm{l}$ of the HotStarTaq Plus Master Mix 2x, $2 \mu$ l of CoralLoad Concentrate $10 \mathrm{x}$, and $0.1 \mu \mathrm{l}$ of each primer (final concentration of $0.5 \mu \mathrm{M}$ ), and it was supplemented with up to $20 \mu \mathrm{l}$ of RNasefree water. The applied reaction conditions included a preliminary denaturation step at $95^{\circ} \mathrm{C}$ for $5 \mathrm{~min}$, followed by 30 cycles of denaturation at $94^{\circ} \mathrm{C}$ for $45 \mathrm{~s}$, annealing at $60^{\circ} \mathrm{C}$ for $60 \mathrm{~s}$, and elongation at $72^{\circ} \mathrm{C}$ for $45 \mathrm{~s}$. The last reaction was followed by extension at $72^{\circ} \mathrm{C}$ for $10 \mathrm{~min}$. Amplicon size was evaluated by comparison with the standard mass of 
GeneRuler 100 bp Ladder Plus (Fermentas UAB, Vilnius, Lithuania). The following amplicons were searched: ail gene fragments with the size of $356 \mathrm{bp}, y s t A$ gene fragments with the size of $134 \mathrm{bp}, y s t B$ gene fragments with the size of $180 \mathrm{bp}$, and $y s t C$ gene fragments with the size of $284 \mathrm{bp}$. The specificity of all amplicons was confirmed by purification with the CleanUp kit (A\&A Biotechnology, Gdynia, Poland) and sequencing (Genomed, Warsaw, Poland).

2.5. Y. enterocolitica Serotype and Biotype Identification. The molecularly confirmed $Y$. enterocolitica strains were serotyped in the slide agglutination test according to a previously described methodology [7] with use of somatic antigens O:3, O:5, O:8, O:9, and O:27 (Sifin, Berlin, Germany). The strain was classified as nonidentified (NI) in the absence of agglutination with any of the sera. The biotype was determined based on pyrazinamidase and Tween esterase activity, esculin hydrolysis, indole production, and salicin, xylose, and trehalose fermentation according to the PN-EN ISO 10273 standard.

2.6. Antimicrobial Susceptibility of the Isolated Y. enterocolitica Strains. Yersinia enterocolitica strains were tested for susceptibility to amoxicillin with $30 \mu \mathrm{g}$ of clavulanic acid, $10 \mu \mathrm{g}$ of ampicillin, $30 \mu \mathrm{g}$ of cefotaxime, $30 \mu \mathrm{g}$ of ceftazidime, $30 \mu \mathrm{g}$ of cefalexin, $5 \mu \mathrm{g}$ of ciprofloxacin, $30 \mu \mathrm{g}$ of chloramphenicol, $10 \mu \mathrm{g}$ of gentamycin, $30 \mu \mathrm{g}$ of kanamycin, $30 \mu \mathrm{g}$ of nalidixic acid, $10 \mu \mathrm{g}$ of streptomycin, sulfamethoxazole/trimethoprim 19:1, and $30 \mu \mathrm{g}$ of tetracycline (Oxoid, Thermo Scientific). These analyses were performed by the standard disc diffusion technique after $24 \mathrm{~h}$ incubation on Mueller-Hinton (Oxoid, Thermo Scientific) agar plates at $30^{\circ} \mathrm{C}$, and they were interpreted according to Clinical and Laboratory Standards Institute (CLSI) guidelines [18].

\section{Results}

Bacteriological examinations of 894 samples collected from 447 birds led to the isolation of 20 strains with biochemical features characteristic of the genus Yersinia. Seven strains were classified as Y. kristensenii, 5 strains as Y. frederiksenii, 7 strains as $Y$. enterocolitica, and 1 strain as Y. frederiksenii/Y. intermedia with the use of API 20E. All 20 strains were molecularly examined, and the genes characteristic of $Y$. enterocolitica were detected in 8 strains. Six strains were biochemically identified as Y. enterocolitica, and the remaining two strains were identified as Y. frederiksenii. One strain, initially classified as a Y. enterocolitica with API 20E, was not molecularly confirmed.

The prevalence of $Y$. enterocolitica in green-winged teals was determined at $1.4 \%$. Two strains were isolated from the samples collected in the Małopolska voivodeship. The strains harboring amplicons of $y s t B$ gene fragments originated from the warm culture (ITC). They belonged to biotype $1 \mathrm{~A}$ and were serotyped as NI (nonidentified) due to the absence of agglutination with any of the analyzed sera. The results of the biochemical and molecular analyses and the biotypes and serotypes of the isolated Y. enterocolitica strains are presented in Table 1.
All Y. enterocolitica strains were resistant to amoxicillin with clavulanic acid, ampicillin, cefalexin, kanamycin, and streptomycin. They were intermediately resistant to cefotaxime, ceftazidime, chloramphenicol, and tetracycline. All $Y$. enterocolitica strains were susceptible only to sulfamethoxazole with trimethoprim, and they differed in susceptibility to the remaining antimicrobials. The antimicrobial susceptibility of $Y$. enterocolitica strains is presented in Table 2 .

The prevalence of Y. enterocolitica in Eurasian coots was determined at $5.0 \%$. Similarly to green-winged teals, one strain was isolated from the sample collected in the Małopolska voivodeship. This strain was $y s t B$-positive, and it originated from the cold culture (PSB) and was bioserotyped as 1A/NI (Table 1). The antimicrobial susceptibility test revealed that the isolated $Y$. enterocolitica strain was resistant to amoxicillin with clavulanic acid, ampicillin, cefalexin, cefotaxime, kanamycin, and streptomycin. The strain was susceptible to nalidixic acid and sulfamethoxazole with trimethoprim, and it was intermediately susceptible to the remaining antibiotics. Detailed characteristics are presented in Table 2 .

The prevalence of $Y$. enterocolitica in capercaillie fecal samples was determined at $4.8 \%$. Five strains were isolated from the fecal material collected in the Wildlife Park in Kadzidłowo, and the prevalence of Y.enterocolitica in these samples reached $62.5 \%$. All strains harbored amplicons whose size corresponded to $y s t B$ gene fragments. Three strains originated from the ITC culture, and 2 strains originated from the PSB culture. Four strains belonged to biotype 1A, and only one strain was identified as biotype 1B. Serotyping revealed that 1 of the 5 isolated $Y$.enterocolitica strains represented the 0:9 serotype, and the remaining strains were not identified. Two Y. enterocolitica strains with different bioserotypes (1A/O:9 and 1A/NI) were isolated from a fecal sample collected from the same female. Detailed results of the biochemical and molecular analyses and the biotypes and serotypes of the isolated Y. enterocolitica strains are presented in Table 1. All Y. enterocolitica strains were resistant to amoxicillin with clavulanic acid, ampicillin, and cefalexin. One strain was additionally resistant to sulfamethoxazole with trimethoprim, and one strain was also resistant to kanamycin. Two of the tested strains were intermediately resistant to chloramphenicol. All Y. enterocolitica strains were susceptible to the remaining antibiotics. The antimicrobial susceptibility of Y. enterocolitica strains is presented in Table 2.

The bacteriological examinations of 36 fecal swabs from black grouse led to the isolation of 1 strain with biochemical features characteristic of the genus Yersinia. This strain was biochemically classified as Y. kristensenii. The molecular analysis confirmed that the strain did not harbor amplicons of yst genes characteristic of Y. enterocolitica.

One strain with biochemical features characteristic of the genus Yersinia was also isolated from white-fronted geese. The molecular analysis confirmed that this strain harbored amplicons of the ail gene. However, the ail gene never occurs alone in $Y$. enterocolitica strains, and it is always accompanied by one of the $y s t$ genes. In biochemical tests involving API 20E, this strain revealed features characteristic of $Y$. frederiksenii/Y. intermedia. 
TABLE 1: Characteristics of Y. enterocolitica strains isolated from free-living birds.

\begin{tabular}{|c|c|c|c|c|c|c|c|c|}
\hline \multirow{2}{*}{ Strain no. } & \multirow{2}{*}{ Bird species } & \multirow{2}{*}{$\begin{array}{c}\text { Biochemical identification } \\
\text { API 20E }\end{array}$} & \multicolumn{4}{|c|}{ Molecular examination } & \multirow{2}{*}{ Molecular identification } & \multirow[b]{2}{*}{ Bioserotype } \\
\hline & & & ail & $y s t A$ & $y s t B$ & $y s t C$ & & \\
\hline $30 \mathrm{PSB}$ & Capercaillie & Y. enterocolitica & - & - & + & - & Y. enterocolitica & $1 \mathrm{~A} / \mathrm{NI}$ \\
\hline 31PSB & Capercaillie & Y. frederiksenii & - & - & + & - & Y. enterocolitica & $1 \mathrm{~A} / \mathrm{NI}$ \\
\hline 35ITC & Capercaillie & Y. frederiksenii & - & - & + & - & Y. enterocolitica & $1 \mathrm{~A} / \mathrm{NI}$ \\
\hline 35PSB & Capercaillie & Y. enterocolitica & - & - & + & - & Y. enterocolitica & 1A/O:9 \\
\hline 36ITC & Capercaillie & Y. enterocolitica & - & - & + & - & Y. enterocolitica & $1 \mathrm{~B} / \mathrm{NI}$ \\
\hline 116ITC & Eurasian coot & Y. enterocolitica & - & - & + & - & Y. enterocolitica & $1 \mathrm{~A} / \mathrm{NI}$ \\
\hline 117ITC & Eurasian coot & Y. enterocolitica & - & - & + & - & Y. enterocolitica & $1 \mathrm{~A} / \mathrm{NI}$ \\
\hline 1GWPB & Green-winged teal & Y. enterocolitica & - & - & + & - & Y. enterocolitica & $1 \mathrm{~A} / \mathrm{NI}$ \\
\hline
\end{tabular}

TABLE 2: Antimicrobial susceptibility of Y. enterocolitica strains isolated from free-living birds.

\begin{tabular}{|c|c|c|c|c|c|c|c|c|}
\hline \multirow[b]{2}{*}{ Antimicrobial } & \multicolumn{8}{|c|}{ Strain no. } \\
\hline & $\begin{array}{c}30 \\
\text { PSB }\end{array}$ & $\begin{array}{c}31 \\
\text { PSB }\end{array}$ & $\begin{array}{c}35 \\
\text { ITC }\end{array}$ & $\begin{array}{c}35 \\
\text { PSB }\end{array}$ & $\begin{array}{c}36 \\
\text { ITC }\end{array}$ & $\begin{array}{l}116 \\
\text { ITC }\end{array}$ & $\begin{array}{l}117 \\
\text { ITC }\end{array}$ & $\begin{array}{c}1 \mathrm{GW} \\
\mathrm{PSB}\end{array}$ \\
\hline Amoxicillin+clavulanic acid $30 \mu \mathrm{g}$ & $\mathrm{R}^{\mathrm{a}}$ & $\mathrm{R}$ & $\mathrm{R}$ & $\mathrm{R}$ & $\mathrm{R}$ & $\mathrm{R}$ & $\mathrm{R}$ & $\mathrm{R}$ \\
\hline Ampicillin $10 \mu \mathrm{g}$ & $\mathrm{R}$ & $\mathrm{R}$ & $\mathrm{R}$ & $\mathrm{R}$ & $\mathrm{R}$ & $\mathrm{R}$ & $\mathrm{R}$ & $\mathrm{R}$ \\
\hline Cefotaxime $30 \mu \mathrm{g}$ & $S^{b}$ & $S$ & S & S & S & I & I & $\mathrm{R}$ \\
\hline Ceftazidime $30 \mu \mathrm{g}$ & S & S & S & S & $S$ & I & I & I \\
\hline Cephalexin $30 \mu \mathrm{g}$ & $\mathrm{R}$ & $\mathrm{R}$ & $\mathrm{R}$ & $\mathrm{R}$ & $\mathrm{R}$ & $\mathrm{R}$ & $\mathrm{R}$ & $\mathrm{R}$ \\
\hline Ciprofloxacin $5 \mu \mathrm{g}$ & $S$ & $S$ & $S$ & S & $S$ & I & $S$ & I \\
\hline Chloramphenicol $30 \mu \mathrm{g}$ & $\mathrm{I}^{\mathrm{c}}$ & S & S & I & S & I & I & I \\
\hline Gentamycin $10 \mu \mathrm{g}$ & $S$ & S & S & S & S & $\mathrm{R}$ & I & I \\
\hline Kanamycin $30 \mu \mathrm{g}$ & S & $\mathrm{R}$ & S & S & S & $\mathrm{R}$ & $\mathrm{R}$ & $\mathrm{R}$ \\
\hline Nalidixic acid $30 \mu \mathrm{g}$ & S & S & S & S & S & $\mathrm{R}$ & I & S \\
\hline Streptomycin $10 \mu \mathrm{g}$ & S & S & S & $S$ & S & $\mathrm{R}$ & $\mathrm{R}$ & $\mathrm{R}$ \\
\hline Sulfamethoxazole/trimethoprim 19:1 & $\mathrm{R}$ & S & S & $S$ & S & $S$ & S & S \\
\hline Tetracycline $30 \mu \mathrm{g}$ & S & S & S & S & S & I & I & I \\
\hline
\end{tabular}

${ }^{a} \mathrm{R}$ : resistant; ${ }^{\mathrm{b}} \mathrm{S}$ : susceptible; ${ }^{\mathrm{c}} \mathrm{I}$ : intermediately resistant.

Strains with biochemical features characteristic of the genus Yersinia were not identified in bacteriological analyses of the samples collected from mallards, pochards, greylag geese, bean geese, and tufted ducks.

\section{Discussion}

Yersinia enterocolitica is one of the most important etiological factors of human diarrhea [4]. Research into the prevalence of $Y$. enterocolitica in various animal species is vital because products of animal origin are the main sources of infection for humans [19]. The prevalence of Y. enterocolitica in wild animals has been studied in Poland, Europe, and in other regions of the world. However, some species of freeliving birds have never been investigated. In Poland, mallards and pheasant were the only bird species to be tested for $Y$. enterocolitica. Molecularly confirmed $Y$. enterocolitica strains were isolated from 5 out of 45 mallards (11.1\%) and were not detected in any of the tested pheasants [7].

In Europe, Levrè et al. [20] examined intestinal loops from 217 birds of 17 species. Thirty-seven strains belonging to the genus Yersinia were isolated from 26 birds. Most of the isolates were identified as Y.enterocolitica (28 strains), 3 isolates as $Y$. frederiksenii, 3 isolates as Y. intermedia, and 1 isolate as $Y$. pseudotuberculosis. However, molecular tests were not conducted. More recently, Foti et al. [21] tested 218 fecal swabs and 21 internal organs from different migratory birds. Eight $Y$. enterocolitica strains (3.35\%) were detected in analyses involving bacteriological examinations and API 20E, but molecular tests for Y. enterocolitica were not carried out.

The prevalence of $Y$. enterocolitica in wild birds was also studied outside Europe. In Japan, Kato et al. [22] detected $Y$. enterocolitica strains in 2 pheasants, 1 domestic pigeon, 1 tree sparrow, 5 crows, 5 blue magpies, 4 bulbuls, and 2 grey starlings, which accounted for $4 \%$ of the tested samples. Shayegani et al. [23] found that $3.3 \%$ of 576 wild birds examined in the USA were positive for $Y$. enterocolitica. Bacterial strains were isolated from 2 great horned owls, 1 Canada goose, 2 wild turkeys, 1 mallard, 1 red-tailed hawk, 1 cowbird, 1 starling, 2 grackles, 1 ring-billed gull, 1 ruffed grouse, 2 redwinged blackbirds, 1 sparrow hawk, 1 canvasback duck, 1 long-eared owl, and 1 wood duck. In Malaysia, Y. enterocolitica strains were isolated from 32 out of 291 (11.0\%) 
examined wild ducks and from 13 out of 180 (7.2\%) examined wild geese [24].

In our study, $Y$. enterocolitica strains were detected in $1.4 \%$ of green-winged teals, $4.8 \%$ of capercaillie, and $5.0 \%$ of Eurasian coots. Our findings do not differ significantly from those reported by other authors, but none of the cited authors relied on molecular methods which are much more accurate and reliable. In our study, the results of the analysis conducted with API 20E did not fully correspond to the results of the molecular test for $Y$. enterocolitica virulence markers. Four strains were identified as Y. enterocolitica using API 20E, but $y s t B$ gene fragments were detected in 3 strains only. However, 2 strains identified as $Y$. frederiksenii with API 20E were molecularly classified as Y. enterocolitica. The above findings indicate that molecular examinations are essential for proper detection and classification of the isolated strains.

Interestingly, the strains identified in the capercaillie were isolated from a single location. The above could be attributed to the fact that $Y$. enterocolitica is easily transmitted. The pathogen is transferred between animals when the carriers are present in the environment [25]. The high percentage of $Y$. enterocolitica strains isolated in the Wildlife Park in Kadzidłowo could result from pathogen transmission in aviaries in breeding centers. The above could pose a threat for humans who come into direct contact with infected birds.

In our study, 1A/NI was the predominant bioserotype. The presence of single $Y$. enterocolitica strains belonging to bioserotypes 1A/O:9 and 1B/NI was also demonstrated. In a study by Kato et al. [22], Y. enterocolitica strains were also classified as biovar 1 (biotype 1) with different serotypes (O:3; O:4; O:4,32; O:5A; O:6,30; O:7,8; and O:14). Shayegani et al. [23] classified 12 out of 19 Y. enterocolitica strains into nine serogroups (O:4,33; O:5,27; O:6,31; O:7,14; O:8,14; $\mathrm{O}: 16 ; \mathrm{O}: 30,31 ; \mathrm{O}: 31$; and $\mathrm{O}: 34)$, and the remaining strains were not identified. In our study, $2 Y$. enterocolitica strains with different bioserotypes were isolated from one fecal sample. Nikolova et al. [26] isolated different $Y$. enterocolitica strains from the same animal, which could indicate that the gastrointestinal tract can be colonized by more than one type of $Y$. enterocolitica strains.

The antimicrobial susceptibility analysis revealed that all tested isolates were resistant to amoxicillin with clavulanic acid, ampicillin, and cefalexin. It should also be noted that the strains isolated from migratory birds (green-winged teals, Eurasian coots) and those obtained from birds living in a specific territory (capercaillie) differed in resistance to chemotherapeutics. The strains isolated from migratory birds were also resistant to kanamycin and streptomycin, and they were resistant and intermediately resistant to cefotaxime, ceftazidime, chloramphenicol, gentamycin, and tetracycline, to which the strains isolated from the capercaillie were susceptible. The fact that these strains were susceptible to only one or two chemotherapeutics gives serious cause for concern because migratory birds use public drinking water sources. Our results cannot be compared with the findings of Foti et al. [21] who studied antimicrobial susceptibility but did not report specific results for Y. enterocolitica strains.

\section{Conclusions}

The presence of $Y$. enterocolitica in green-winged teals, Eurasian coots, and capercaillie indicates that these birds could be carriers, potential reservoirs, and sources of infection for humans. These birds can also be regarded as reliable bioindicators of $Y$. enterocolitica in their respective habitats.

\section{Data Availability}

The data used to support the findings of this study are available from the corresponding author upon request.

\section{Conflicts of Interest}

The authors declare that they have no competing interests.

\section{Acknowledgments}

This study was funded by the KNOW (Leading National Research Centre) Scientific Consortium "Healthy AnimalSafe Food," decision of Ministry of Science and Higher Education No. 05-1/KNOW2/2015. This publication was funded by the Minister of Science and Higher Education in the range of the program entitled "Regional Initiative of Excellence" for the years 2019-2022, Project No. 010/RID/2018/19, amount of funding 12,000,000 PLN.

\section{References}

[1] T. Strzała, A. Kowalczyk, and E. Łukaszewicz, "Reintroduction of the European capercaillie from the capercaillie breeding centre in Wisła Forest District: genetic assessments of captive and reintroduced populations," PLoS One, vol. 10, no. 12, article e0145433, 2015.

[2] E. J. Bottone, "Yersinia enterocolitica: Revisitation of an Enduring Human Pathogen," Clinical Microbiology Newsletter, vol. 37, no. 1, pp. 1-8, 2015.

[3] A. McNally, N. R. Thomson, S. Reuter, and B. W. Wren, "'Add, stir and reduce': Yersinia spp. as model bacteria for pathogen evolution," Nature Reviews Microbiology, vol. 14, no. 3, pp. 177-190, 2016.

[4] EFSA (European Food Safety Authority and European Centre for Disease Prevention and Control), "EU summary report on zoonoses, zoonotic agents and food-borne outbreaks 2015," EFSA Journal, vol. 14, pp. 4634-4865, 2016.

[5] M. Bucher, C. Meyer, B. Grötzbach, S. Wacheck, A. Stolle, and M. Fredriksson-Ahomaa, "Epidemiological data on pathogenic Yersinia enterocolitica in southern Germany during 2000-2006," Foodborne Pathogens and Disease, vol. 5, no. 3, pp. 273-280, 2008.

[6] E. Huovinen, L. M. Sihvonen, M. J. Virtanen, K. Haukka, A. Siitonen, and M. Kuusi, "Symptoms and sources of Yersinia enterocolitica infection: a case-control study," BMC Infectious Diseases, vol. 10, no. 1, p. 122, 2010.

[7] A. Bancerz-Kisiel, A. Szczerba-Turek, A. Platt-Samoraj, P. Socha, and W. Szweda, "Application of multiplex PCR for the evaluation of the occurrence of ail, $y s t A$ and $y s t B$ genes in Yersinia enterocolitica strains isolated from wild boars (Sus scrofa)," Bulletin of the Veterinary Institute in Pulawy, vol. 53, pp. 351-355, 2009. 
[8] K. Syczyło, A. Platt-Samoraj, A. Bancerz-Kisiel et al., "The prevalence of Yersinia enterocolitica in game animals in Poland," PLoS One, vol. 13, no. 3, article e0195136, 2018.

[9] K. Morka, G. Cieniuch, and G. Bugla-Płoskońska, "Epidemiology of Yersinia enterocolitica with special consideration of animal reservoir," Postępy Higieny i Medycyny Doświadczalnej, vol. 72, pp. 594-605, 2018.

[10] A. Bancerz-Kisiel, A. Szczerba-Turek, K. Lipczyńska, T. Stenzel, and W. Szweda, "Bioserotypes and virulence markers of Yersinia enterocolitica strains isolated from mallards (Anas platyrhynchos) and pheasants (Phasianus colchicus)," Journal of Food Protection, vol. 75, no. 12, pp. 22192222, 2012.

[11] N. Bhagat and J. S. Virdi, "Distribution of virulence-associated genes in Yersinia enterocolitica biovar 1A correlates with clonal groups and not the source of isolation," FEMS Microbiology Letters, vol. 266, no. 2, pp. 177-183, 2007.

[12] A. Platt-Samoraj, A. Bancerz-Kisiel, and W. Szweda, "Yersinia enterocolitica pathogenicity and the significance of biotype $1 \mathrm{~A}$ in the pathogenesis of yersiniosis," Medycyna Weterynaryjna, vol. 62, pp. 1113-1115, 2006.

[13] J. Batzilla, J. Heesemann, and A. Rakin, "The pathogenic potential of Yersinia enterocolitica 1A," International Journal of Medical Microbiology, vol. 301, no. 7, pp. 556-561, 2011.

[14] A. McNally, T. Dalton, R. M. La Ragione, K. Stapleton, G. Manning, and D. G. Newell, "Yersinia enterocolitica isolates of differing biotypes from humans and animals are adherent, invasive and persist in macrophages, but differ in cytokine secretion profiles in vitro," Journal of Medical Microbiology, vol. 55, Part 12, pp. 1725-1734, 2006.

[15] T. Ramamurthy, K. I. Yoshino, X. Huang et al., "The novel heat-stable enterotoxin subtype gene $(y s t B)$ of Yersinia enterocolitica: nucleotide sequence and distribution of the $y$ st genes," Microbial Pathogenesis, vol. 23, no. 4, pp. 189-200, 1997.

[16] I. Singh and J. S. Virdi, "Production of Yersinia stable toxin (YST) and distribution of yst genes in biotype 1A strains of Yersinia enterocolitica," Journal of Medical Microbiology, vol. 53, no. 11, pp. 1065-1068, 2004.

[17] A. Bancerz-Kisiel, A. Szczerba-Turek, A. Platt-Samoraj, and W. Szweda, "Application of multiplex PCR for the evaluation of the occurrence of $y s t A, y s t B, y s t C$ and $y m o A$ genes in Yersinia enterocolitica strains isolated from fattening pigs," Bulletin of the Veterinary Institute in Pulawy, vol. 55, pp. 33-37, 2011.

[18] CLSI (Clinical and Laboratory Standards Institute), "Performance standards for antimicrobial susceptibility testing; twenty third informational supplement. Tech. Rep. M100S23," CLSI, Wayne, Pa, USA, 2013.

[19] M. Fredriksson-Ahomaa, A. Stolle, and H. Korkeala, "Molecular epidemiology of Yersinia enterocolitica infections," FEMS Immunology and Medical Microbiology, vol. 47, no. 3, pp. 315-329, 2006.

[20] E. Levrè, P. Valentini, M. Brunetti, and F. Sacchelli, "Stationary and migratory avifauna as reservoirs of Salmonella, Yersinia and Campylobacter," Annali di Igiene, vol. 1, no. 3-4, pp. 729-740, 1989.

[21] M. Foti, D. Rinaldo, A. Guercio et al., "Pathogenic microorganisms carried by migratory birds passing through the territory of the island of Ustica, Sicily (Italy)," Avian Pathology, vol. 40, no. 4, pp. 405-409, 2011.

[22] Y. Kato, K. Ito, Y. Kubokura, T. Maruyama, K. Kaneko, and M. Ogawa, "Occurrence of Yersinia enterocolitica in wild- living birds and Japanese serows," Applied and Environmental Microbiology, vol. 49, no. 1, pp. 198-200, 1985.

[23] M. Shayegani, W. B. Stone, I. DeForge, T. Root, L. M. Parsons, and P. Maupin, "Yersinia enterocolitica and related species isolated from wildlife in New York State," Applied and Environmental Microbiology, vol. 52, no. 3, pp. 420-424, 1986.

[24] H. Jamali, B. Radmehr, and S. Ismail, "Prevalence and antimicrobial resistance of Listeria, Salmonella, and Yersinia species isolates in ducks and geese," Poultry Science, vol. 93, no. 4, pp. 1023-1030, 2014.

[25] S. Virtanen, L. Salonen, R. Laukkanen-Ninios, M. FredrikssonAhomaa, and H. Korkeala, "Piglets are a source of pathogenic Yersinia enterocolitica on fattening-pig farms," Applied and Environmental Microbiology, vol. 78, no. 8, pp. 3000-3003, 2012.

[26] S. Nikolova, Y. Tzvetkov, H. Najdenski, and A. Vesselinova, "Isolation of pathogenic Yersiniae from wild animals in Bulgaria," Journal of Veterinary Medicine Series B, vol. 48, no. 3, pp. 203-209, 2001. 